\title{
The first factor of the class number of the $p$-th cyclotomic field
}

\author{
Korneel Debaene
}

\begin{abstract}
Kummer's conjecture states that the relative class number of the $p$-th cyclotomic field follows a strict asymptotic law. Granville has shown it unlikely to be true - it cannot be true if we assume the truth of two other widely believed conjectures. We establish a new bound for the error term in Kummer's conjecture, and more precisely we prove that $\log \left(h_{p}^{-}\right)=\frac{p+3}{4} \log p+\frac{p}{2} \log (2 \pi)+\log (1-\beta)+O\left(\log _{2} p\right)$, where $\beta$ is a possible Siegel zero of an $L(s, \chi), \chi$ odd.
\end{abstract}

Mathematics Subject Classification (2010). Primary 11R18; Secondary 11R29.

Keywords. Class numbers, Relative Class numbers, Cyclotomic Fields, Kummer's Conjecture, $L$-functions.

\section{Introduction}

Let $h_{p}$ denote the class number of $\mathbb{Q}\left(\zeta_{p}\right)$, where $p$ is an odd prime. Let $h_{p}^{+}$ denote the class number of the totally real field $\mathbb{Q}\left(\zeta_{p}+\zeta_{p}^{-1}\right)$. It is well known that $h_{p}^{+}$divides $h_{p}$. We denote the quotient - or so called first factor of $h_{p}$ - by $h_{p}^{-}$. The following formula is an application of the class number formula(see e.g. [9])

$$
h_{p}^{-}=G(p) \prod_{\chi \bmod p, \text { odd }} L(1, \chi),
$$

where $G(p)=2 p\left(\frac{p}{4 \pi^{2}}\right)^{\frac{p-1}{4}}$. Since the magnitude of the $L(1, \chi)$ is not evident, it is hoped they are insignificant. The guess that $h_{p}^{-}$is asymptotically equivalent to $G(p)$ is known as Kummer's Conjecture. It is opportune to study the logarithm of this equation because the orthogonality property of characters 
gives us

$$
\sum_{\chi \bmod p, \text { odd }} \log (L(s, \chi))=\frac{p-1}{2}\left(\sum_{q^{m} \equiv 1(p)} \frac{1}{m q^{m s}}-\sum_{q^{m} \equiv-1(p)} \frac{1}{m q^{m s}}\right) .
$$

One can estimate this sum to the right of $s=1$, where good estimates are available, and using a zero-free region of the $L$-functions, one can bound the derivative in a neighbourhood of $s=1$. Masley and Montgomery [5] obtained with these key ingredients that $\left|\log \left(h_{p}^{-} / G(p)\right)\right|<7 \log p$ for $p>200$, which is strong enough to solve the class number one problem for cyclotomic fields.

Puchta [8] improved this approach by using analogous bounds on higher derivatives, and using a near zero-free region, namely the open ball $B\left(1, \frac{1}{c \log p}\right)$ with center 1 and radius $\frac{1}{c \log p}$, where $c$ is some big enough constant. This is a zerofree region for all but possibly one $L$-function $\bmod p$, which then is necessarily quadratic and has one zero $\beta$ in this region, which is necessarily real and simple and goes by the name of a Siegel zero. It is worth mentioning that if $p=1 \bmod 4$, the odd characters are not quadratic, hence have no Siegel zero. Puchta obtained $\log \left(h_{p}^{-} / G(p)\right)=\log (1-\beta)+O\left(\left(\log _{2} p\right)^{2}\right)$.

Our proof will follow the main ideas from [8], but our practical implementation in section 3 is of a different nature, and yields

Theorem 1.1. If no Siegel zero is present among the odd Dirichlet L-functions of conductor $p$, then the relative class number of $\mathbb{Q}\left(\zeta_{p}\right)$ satisfies

$$
\left|\log \left(h_{p}^{-} / G(p)\right)\right| \leq 2 \log _{2}(p)+O\left(\log _{3}(p)\right)
$$

If there is a Siegel zero $\beta$ present among the odd Dirichlet L-functions of conductor $p$, then the relative class number of $\mathbb{Q}\left(\zeta_{p}\right)$ satisfies

$$
\left|\log \left(h_{p}^{-} / G(p)\right)-\log (1-\beta)\right| \leq 4 \log _{2}(p)+O\left(\log _{3}(p)\right)
$$

Since $\log (1-\beta)$ is negative, an upper bound without this term may be deduced. Finally, we note that this result sharpens the best known estimate, by Lepistö [3]. Indeed, he proves an upper bound for $\log \left(h_{p}^{-} / G(p)\right)$ with main term $5 \log _{2}(p)$.

\section{Bounds around $s=1$}

In this section we exploit formula (1.2), which gives a representation in terms of splitting behaviour in $\mathbb{Q}\left(\zeta_{p}\right) / \mathbb{Q}$. We define

$$
\Pi(x, p, a)=\sum_{q^{m} \leq x, q^{m} \equiv a(p)} \frac{1}{m q^{m}},
$$

where $q^{m}$ ranges over the primepowers. A Brun-Titschmarsh style bound is given by the following lemma.

Lemma 2.1. For $x>p$, and $p>500$ we have that

$$
\Pi(x, p, \pm 1) \leq \frac{2 x}{(p-1) \log (x / p)}
$$


Proof. When $x \geq p^{2}$, we start from the following inequality (see [5], Lemma 1)

$$
\Pi(x, p, \pm 1) \leq \pi(x, p, \pm 1)+\frac{4 \sqrt{x}}{p}+\log x .
$$

In [7], the following strong version of the Brun-Titchmarsh inequality is proven.

$$
\pi(x, p, \pm 1) \leq \frac{2 x}{(p-1)(\log (x / p)+5 / 6)}
$$

Thus we only need to prove that

$$
\frac{4 \sqrt{x}}{p}+\log x<\frac{2 x}{(p-1)}\left(\frac{1}{\log (x / p)}-\frac{1}{\log (x / p)+5 / 6}\right) .
$$

By setting $x=p X, X \geq p$, it suffices to prove that

$$
g(X):=\frac{4}{\sqrt{p}}+\frac{\log (p X)}{\sqrt{X}}<h(X):=\frac{5 \sqrt{X}}{3(\log X+5 / 6)^{2}} .
$$

Now, $g(X)$ decreases for $X-e^{2}$ and $h(X)$ increases for $X \geq e^{19 / 6}$, hence it suffices to check that

$$
g(p)=\frac{4}{\sqrt{p}}+\frac{2 \log (p)}{\sqrt{p}}<h(p)=\frac{5 \sqrt{p}}{3(\log p+5 / 6)^{2}}
$$

for $p \geq 500$. Now, $g(p)$ decreases for $p \geq 2$ and $h(p)$ increases for $p \geq e^{19 / 6}$, hence it suffices to check that $g(500)<h(500)$, which is clear.

When $p<x<p^{2}$, any two primepowers in the sum $\Pi(x, p, \pm 1)$ are necessarily coprime. Indeed, their quotient would be $1 \bmod p$, so at least $p+1$, implying that the smallest one should be less than $\frac{p^{2}}{p+1}$. The only option then is that $p-1=2^{m}$ and $p^{2}-1=2^{k}$, but except for $p=3$ this is impossible. Thus, $\Pi(x, p, \pm 1) \leq N(x, Q, p, \pm 1)+\pi(Q)$, where $N(x, Q, p, a)$ is the number of integers $n \equiv a(\bmod p), n \leq x$ such that $n$ is not divisible by any prime number less then $Q$. We may bound $\pi(Q)$ trivially by $Q$, so that the quantity to be bounded is $N(x, Q, p, \pm 1)+Q$.

In the proof of the Brun-Titchmarsh inequality

$$
\pi(x, q, \pm 1) \leq \frac{2 x}{(p-1) \log (x / p)}
$$

using the large sieve, as in [6, p.42-44], the first step is to bound $\pi(x, q, \pm 1)$ by exactly the quantity $N(x, Q, p, \pm 1)+Q$. This shows that in this range of $x$, the large sieve method for the Brun-Titchmarsh inequality can be applied with the same success for primepowers as for primes.

Let us define $f(s)$ by

$$
f(s)=\left(\sum_{\chi(-1)=-1} \log L(s, \chi)\right)-\log (s-\beta),
$$

in case that any of the $L$-functions with $\chi$ odd has a so-called Siegel zero $\beta$ in ] $\left.1-\frac{1}{c \log p}, 1\right]$, where $c$ is some big enough constant. Otherwise, we leave out the term with the Siegel zero. In any case $f$ is holomorphic in $B\left(1, \frac{1}{c \log p}\right)$. 
Lemma 2.2. For any $c, p \geq 500$, and $\left.\sigma \in] 1,1+\frac{1}{c \log p}\right]$, we have the following estimates.

$$
\begin{aligned}
& |f(\sigma)| \leq\left(1+1_{\beta}\right) \log \left(\frac{1}{\sigma-1}\right)+\frac{3}{2} \\
& \left|f^{(\nu)}(\sigma)\right| \leq\left(1+1_{\beta}+c_{p, \nu}\right) \frac{(\nu-1) !}{(\sigma-1)^{\nu}}
\end{aligned}
$$

Where the notation $1_{\beta}$ stands for 1 if a Siegel zero is present and 0 otherwise, and we may choose the $c_{p, \nu}$ to be equal to $\frac{\log (2)}{2 c^{\nu}(\nu-1) ! \log p}+\frac{\log _{2}(p)+\log (c)-\log _{2}(2)+e^{-1}}{c^{\nu}(\nu-1) !}+$ $\frac{1}{c \log p}+\frac{\sigma\lfloor\log \nu\rfloor}{\nu-\lfloor\log \nu\rfloor}+\frac{\sigma \nu}{c^{\lfloor\log \nu\rfloor\lfloor\log \nu\rfloor !} .}$

Proof. The case $\nu=0$ can be proven as in [5]. The estimates for the derivatives are stated in [8], but the statement is slightly incorrect and the proof omitted, so we will prove them here in full. We bound the sums occurring in the $\nu$-th derivative of (1.2) using Lemma 2.1 and partial summation.

$$
\begin{aligned}
\frac{p-1}{2} & \sum_{q^{m} \equiv 1(p)} \frac{(m \log q)^{\nu}}{m q^{m \sigma}}=\frac{p-1}{2} \int_{2 p}^{\infty} \frac{(\log x)^{\nu} d(\Pi(x, p, 1))}{x^{\sigma}} \\
= & \frac{p-1}{2} \int_{2 p}^{\infty} \frac{\sigma x^{\sigma-1}(\log x)^{\nu}-\nu x^{\sigma-1}(\log x)^{\nu-1}}{x^{2 \sigma}} \Pi(x, p, 1) d x \\
\leq & \int_{2 p}^{\infty} \frac{\sigma(\log x)^{\nu}}{x^{\sigma} \log (x / p)} d x \\
= & \frac{p \sigma}{p^{\sigma}} \int_{2}^{\infty} \frac{(\log x+\log p)^{\nu}}{x^{\sigma} \log x} d x=: I,
\end{aligned}
$$

where we possibly omitted the first term $\frac{(p-1) \log (p+1)^{\nu}}{2 m(p+1)^{\sigma}}$ if $p+1$ is a primepower $q^{m}$. If this is the case, then $q=2$ and $m=\log (p+1) / \log (2)$. This term is smaller than $\varepsilon_{1} \frac{(\nu-1) !}{(\sigma-1)^{\nu}}$ for all $\sigma$ in the desired range for $\varepsilon_{1}=\frac{\log (2)}{2 c^{\nu}(\nu-1) ! \log p}$. We expand the integrand with the binomial theorem, and get

$$
\begin{aligned}
I & =\frac{p \sigma}{p^{\sigma}}(\log p)^{\nu} \int_{2}^{\infty} \frac{1}{x^{\sigma} \log x} d x+\frac{p \sigma}{p^{\sigma}} \sum_{i=0}^{\nu-1} \frac{\nu !(\log p)^{i}}{(\nu-i) ! i !} \int_{1}^{\infty} \frac{(\log x)^{\nu-i-1}}{x^{\sigma}} d x \\
& =\frac{p \sigma}{p^{\sigma}}(\log p)^{\nu} \int_{2}^{\infty} \frac{1}{x^{\sigma} \log x} d x+\frac{(\nu-1) !}{(\sigma-1)^{\nu}} \frac{p \sigma}{p^{\sigma}} \sum_{i=0}^{\nu-1} \frac{\nu}{\nu-i} \frac{((\sigma-1) \log p)^{i}}{i !}
\end{aligned}
$$

where we have used the identity

$$
\int_{1}^{\infty} \frac{(\log x)^{a}}{x^{\sigma}} d x=\int_{0}^{\infty} \frac{t^{a}}{e^{(\sigma-1) t}} d t=\frac{a !}{(\sigma-1)^{a+1}}
$$


We consider first the term

$$
\begin{aligned}
\frac{p \sigma}{p^{\sigma}}(\log p)^{\nu} \int_{2}^{\infty} \frac{1}{x^{\sigma} \log x} d x & =\frac{p \sigma}{p^{\sigma}}(\log p)^{\nu} \int_{\log 2}^{\infty} e^{-(\sigma-1) t} \frac{d t}{t} \\
& \leq \frac{p \sigma}{p^{\sigma}}(\log p)^{\nu}\left(\int_{(\sigma-1) \log 2}^{1} \frac{1}{t} d t+\int_{1}^{\infty} e^{-t} d t\right) \\
& \leq(\log p)^{\nu}\left(\log \left(\frac{1}{\sigma-1}\right)-\log _{2}(2)+e^{-1}\right)
\end{aligned}
$$

Because $p \sigma \leq p^{\sigma}$. We now seek the $\varepsilon_{2}$ such that

$$
(\log p)^{\nu}\left(\log \left(\frac{1}{\sigma-1}\right)-\log _{2}(2)+e^{-1}\right) \leq \varepsilon_{2} \frac{(\nu-1) !}{(\sigma-1)^{\nu}} .
$$

If we put $\varepsilon_{2}=\frac{\log _{2}(p)+\log (c)-\log _{2}(2)+e^{-1}}{c^{\nu}(\nu-1) !}$, the inequality holds for for $\sigma \rightarrow 1$ and for $\sigma=1+\frac{1}{c \log p}$. One may check that the derivative of the difference does not have a zero in the interval under consideration if $p>e^{e}$. Thus the difference is monotone, and the inequality holds throughout.

To deal with the rest of the terms efficiently, write $X=(\sigma-1) \log p \leq$ $1 / c$. Then we have for any integer $B \geq 1$

$$
\begin{aligned}
\frac{p \sigma}{p^{\sigma}} \sum_{i=0}^{\nu-1} \frac{\nu}{\nu-i} \frac{X^{i}}{i !} & \leq \frac{p \sigma}{p^{\sigma}} \sum_{i=0}^{B-1} \frac{\nu}{\nu-B} \frac{X^{i}}{i !}+\frac{p \sigma}{p^{\sigma}} X^{B} \sum_{i=0}^{\nu-1} \frac{\nu}{B !} \frac{X^{i-B}}{(i-B) !} \\
& \leq \frac{p \sigma}{p^{\sigma}} \frac{\nu}{\nu-B} e^{X}+\frac{p \sigma}{p^{\sigma}} \frac{\nu}{c^{\nu} B !} e^{X}=\frac{\nu \sigma}{\nu-B}+\frac{\nu \sigma}{c^{B} B !}
\end{aligned}
$$

We now put $B=\lfloor\log \nu\rfloor$, and see that the to be bounded sum is bounded by $\left(1+\varepsilon_{3}\right) \frac{(\nu-1) !}{(\sigma-1)^{\nu}}$, where $\varepsilon_{3}=\frac{1}{c \log p}+\frac{\sigma\lfloor\log \nu\rfloor}{\nu-\lfloor\log \nu\rfloor}+\frac{\sigma \nu}{c^{\lfloor\log \nu\rfloor\lfloor\log \nu\rfloor !}}$

One may now bound the $\varepsilon_{1}+\varepsilon_{2}+\varepsilon_{3}$ by the coefficient of $\frac{(\nu-1) !}{(\sigma-1)^{\nu}}$ except the $1_{\beta}$ in the statement of the lemma. We note that the sum over the primepowers congruent to $-1 \bmod p$ obeys the same bound with the same proof as above. One of the sums is strictly positive and the other is strictly negative, thus we have proven that

$$
\left|f^{\nu}(s)+(\log (\sigma-\beta))^{(\nu)}\right| \leq\left(1+c_{p, \nu}\right) \frac{(\nu-1) !}{(\sigma-1)^{\nu}}
$$

or since $\frac{(\nu-1) !}{(\sigma-\beta)^{\nu}} \leq \frac{(\nu-1) !}{(\sigma-1)^{\nu}}$

$$
\left|f^{\nu}(s)\right| \leq\left(1+1_{\beta}+c_{p, \nu}\right) \frac{(\nu-1) !}{(\sigma-1)^{\nu}} .
$$

On the other hand we can prove the following bound on the derivatives of $f$ to the right of $s=1$, using the holomorphic property of $f$ on $B\left(1, \frac{1}{c \log p}\right)$, when $c$ is big enough. We note that due to Kadiri ([2], Theorem 12.1) the value $c=6.4355$ is big enough. 
Lemma 2.3. For $c>6.4355, \frac{p-1}{\log p}>c$, and $\sigma \in\left[1,1+\frac{2}{c \log p}\right]$, we have that

$$
\left|f^{(\nu)}(\sigma)\right| \leq 2 c^{\nu} \nu ! p \log ^{\nu+1} p
$$

Proof. Recall the lemma of Borel-Caratheodory (see [1], p. 12) which states that if $g$ is holomorphic and $\Re(g(s)) \leq M$ in $B\left(\sigma_{0}, R\right)$ and $g\left(\sigma_{0}\right)=0$, then

$$
\left|g^{\nu}(s)\right| \leq \frac{2 M \nu !}{(R-r)^{\nu}}, \quad s \in B\left(\sigma_{0}, r\right)
$$

We wish to apply this to $f(s)-f\left(\sigma_{0}\right)$. This function vanishes at $\sigma_{0}$, and is holomorphic as long as $R \leq \sigma_{0}-\left(1-\frac{1}{c \log p}\right)$. For the bound on the real part, consider

$$
L(s, \chi)=\sum_{n=1}^{\infty} \frac{\chi(n)}{n^{s}}=s \int_{1}^{\infty} \frac{\sum_{n \leq x} \chi(n)}{x^{s+1}} d x .
$$

Since $\left|\sum_{n=1}^{x} \chi(n)\right| \leq \frac{p}{2}$, we have that $|L(s, \chi)| \leq|s| \int_{1}^{\infty} \frac{\left|\sum_{n \leq x} \chi(n)\right|}{x^{\sigma+1}} d x \leq \frac{|s| p}{2 \sigma}$. This means that

$$
\Re(f(s)) \leq \frac{p-1}{2}(\log p+\log (|s| / 2 \sigma))-\log (|s-\beta|) .
$$

For $s$ on the border of the domain determined by $3 / 4<\Re(s)<2,|\Im(s)| \leq \frac{1}{4}$, $|s| / 2 \sigma \leq \sqrt{10} / 6$ and say $|s-\beta|>1 / 8$, thus this bound is smaller than $\frac{p-1}{2} \log p$. Since $f(s)$ is harmonic with at most logarithmic singularities in which $\Re(f) \rightarrow-\infty$, the same bound holds also inside the domain. In the region $\sigma>1$, consider the following estimation.

$$
|\Re(\log L(s, \chi))|=\left|\Re\left(\sum_{q^{m}} \frac{\chi\left(q^{m}\right)}{m q^{m s}}\right)\right| \leq \sum_{q^{m}} \frac{1}{m q^{m s}}=\log \zeta(\sigma) \leq \log \left(\frac{\sigma}{\sigma-1}\right),
$$

thus if $\sigma_{0}>p /(p-1)$, then $\left|\Re\left(f\left(\sigma_{0}\right)\right)\right| \leq \frac{p-1}{2} \log (p)+\log (p-1)$. In conclusion, as long as $\sigma_{0}>p /(p-1)$,

$$
\Re\left(f(\sigma)-f\left(\sigma_{0}\right)\right) \leq p \log p .
$$

One retrieves the statement of the theorem by putting $\sigma_{0}=1+\frac{1}{c \log p}, R=$ $\frac{2}{c \log p}, r=\frac{1}{c \log p}$.

\section{Worst case scenario}

Among all functions that satisfy the bounds from the preceding section, what is the largest value $f(1)$ can attain? We define $\sigma_{\nu}$ to be the point where the bound (2.2) and the absolute bound (2.3) coincide. We note that

$$
\sigma_{\nu}-1=\frac{1}{c \log p} \sqrt[\nu]{\frac{1+1_{\beta}+c_{p, \nu}}{2 \nu p \log p}} \geq \frac{1}{c \log p \sqrt[\nu]{2 \nu p \log p}} .
$$


Theorem 3.1. For all $p>500$, and $c>6.4355$,

$$
|f(1)| \leq\left(1+1_{\beta} .2+e^{1 / c}\right) \log _{2}(p)+O(1),
$$

where the $O(1)$-term is bounded by $\left(3+e^{1 / c}\right) \log (c)+0.791 e^{1 / c}+10.720+\frac{0.943}{c}$

Proof. We use the Taylor expansion of $f$ with error term in integral form,

$$
\begin{aligned}
f(1)= & f\left(\sigma_{\nu}\right)+\left(1-\sigma_{\nu}\right) f^{\prime}\left(\sigma_{\nu}\right)+\frac{\left(1-\sigma_{\nu}\right)^{2}}{2} f^{(2)}\left(\sigma_{\nu}\right)+\ldots \\
& +\int_{\sigma_{\nu}}^{1} \frac{f^{(\nu)}(x)}{(\nu-1) !}(1-x)^{\nu-1} d x
\end{aligned}
$$

Now note that $\left|f^{(\nu)}(x)\right|$ is bounded above by the bound (2.3) for all $x$ between 1 and $\sigma_{\nu}$, which is equal to $\left|f^{(\nu)}\left(\sigma_{\nu}\right)\right|$. Using (2.1), (2.2) and (3.1), we get

$$
\begin{aligned}
|f(1)| & \leq\left|f\left(\sigma_{\nu}\right)\right|+\sum_{i=1}^{\nu} \frac{\left(\sigma_{\nu}-1\right)^{i}}{i !}\left|f^{(i)}\left(\sigma_{\nu}\right)\right| . \\
& \leq\left(1+1_{\beta}\right) \log \left(\frac{1}{\sigma_{\nu}-1}\right)+3 / 2+\sum_{i=1}^{\nu} \frac{1+1_{\beta}+c_{p, i}}{i} \\
& \leq\left(1+1_{\beta}\right)\left(\log _{2}(p)+\log (c)+\frac{\log (2 \nu p \log p)}{\nu}\right)+3 / 2+\sum_{i=1}^{\nu} \frac{1+1_{\beta}+c_{p, i}}{i} .
\end{aligned}
$$

Upon taking $\nu=\log p$, this first contribution is bounded by

$$
\left(1+1_{\beta}\right)\left(\log _{2}(p)+\log (c)+1+\frac{\log \left(2(\log p)^{2}\right)}{\log p}\right)+3 / 2 .
$$

In the rest of the terms, we find the first $\nu$ terms of some converging series;

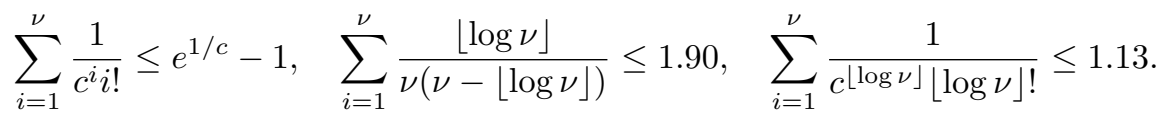

Using this and the well-known estimate $\sum_{i=1}^{\nu} \frac{1}{i} \leq \log (\nu)+1$ we bound the last contribution as follows

$$
\begin{aligned}
\sum_{i=1}^{\nu} \frac{1+1_{\beta}+c_{p, i}}{i} & \leq\left(1+1_{\beta}+\frac{1}{c \log p}\right)(\log (\nu)+1)+\left(1+\frac{1}{c \log p}\right) 3.03 \\
& +\left(\frac{\log (2)}{2 \log p}+\log _{2}(p)+\log (c)-\log _{2}(2)+e^{-1}\right)\left(e^{1 / c}-1\right) .
\end{aligned}
$$

Gathering everything and filling in $p=500$ for the terms converging to zero, we recover the statement of the theorem.

To finish the proof of Theorem 1.1, one now needs to plug the above estimate of $f(1)$ into the logarithm of the formula (1.1), and check that the choice of $c=\log _{2}(p) \frac{6.4355}{\log _{2}(500)}$ is permitted.

Remark 3.2. It is quite counterintuitive that a bigger value of $c$ gives a better estimate in Theorem 3.1 while a smaller value of $c$ means a bigger zero-free region, thus means a stronger input. In truth there is a tradeoff between 
having $\sigma_{\nu}$ big to control the main term coming from Lemma 2.2 and at the same time not too big to bound the term coming from $\varepsilon_{1}$ in the proof of Lemma 2.2. This $\varepsilon_{1}$ cannot be efficiently bounded by a lack of good bounds on the number of primes of the form $a p+1$, where $a$ is a small integer.

Remark 3.3. From (1.1) it is now clear that the general behaviour of $h_{p}^{-}$is dominated by $G(p)$ and that the $L$-values can perturb this term only slightly. It is somewhat common(see [4]) to state upper bounds for $h_{p}^{-}$in terms of $G(p)$, where $4 \pi^{2}$ is replaced by a smaller constant.

Corollary 3.4. We have that $h_{p}^{-} \leq 2 p\left(\frac{p}{39}\right)^{\frac{p-1}{4}}$, for $p>9649$.

Proof. This follows from plugging in $c=\frac{6.4355 \log _{2}(p)}{\log _{2}(500)}=3.523 \log _{2}(p)$ in Theorem 3.1 and checking that

$$
|f(1)| \leq e^{\frac{p-1}{4}} \log \left(\frac{4 \pi^{2}}{39}\right),
$$

whenever $p>9649$.

\section{References}

[1] T. Estermann. Introduction to Modern Prime Number Theory, volume 41 of Cambridge Tracts in Mathematics and Mathematical Physics. Cambridge University Press, 1961.

[2] H. Kadiri. Régions explicites sans zéros pour les fonctions 1 de dirichlet, phd thesis.

[3] Timo Lepistö. On the growth of the first factor of the class number of the prime cyclotomic field. Ann. Acad. Sci. Fenn. Ser. A I, (577):21, 1974.

[4] Stéphane R. Louboutin. Mean values of $L$-functions and relative class numbers of cyclotomic fields. Publ. Math. Debrecen, 78(3-4):647-658, 2011.

[5] J. Myron Masley and Hugh L. Montgomery. Cyclotomic fields with unique factorization. J. Reine Angew. Math., 286/287:248-256, 1976.

[6] H. L. Montgomery. Topics in Multiplicative Number Theory, volume 227 of Lecture Notes in Mathematics. Springer-Verlag, 1971.

[7] H. L. Montgomery and R. C. Vaughan. The large sieve. Mathematika, 20:119134, 1973.

[8] Jan-Christoph Puchta. On the class number of $p$-th cyclotomic field. Arch. Math. (Basel), 74(4):266-268, 2000.

[9] L. C. Washington. Introduction to Cyclotomic Fields, volume 83 of Graduate Texts in Mathematics. Springer, 1982.

Korneel Debaene

Krijgslaan 281, S22

9000 Ghent

Belgium

e-mail: kdbaene@cage.ugent.be 